\title{
The azimuth-dependent offset-midpoint traveltime pyramid in 3D HTI media
}

\author{
Qi Hao*, Alexey Stovas,NTNU Norway, and Tariq Alkhalifah, KAUST Saudi Arabia.
}

\section{Summary}

Analytical representation of offset-midpoint traveltime equation is very important for pre-stack Kirchhoff migration and velocity inversion in anisotropic media. For VTI media, the offset-midpoint traveltime resembles the shape of Cheop's pyramid. In this study, we extend the offset-midpoint traveltime pyramid to the case of 3D HTI media. We employ the stationary phase method to derive the analytical representation of traveltime equation, and then use Shanks transformation to improve the accuracy of horizontal and vertical slownesses. The traveltime pyramid is derived in both the depth- and time-domain. Numerical examples indicate that the azimuthal characteristics of both the traveltime pyramid and the migration isochrones are very obvious in HTI media due to the effect of anisotropy.

\section{Introduction}

The azimuthal characteristics of seismic waves in HTI media can help to identify the azimuth of fractures, which is important for oil and gas exploration in fractured reservoirs. The azimuthal variation of velocities influences amplitudes, but more detectable, traveltimes. Developing analytical prestack traveltime formulations helps in many applications, including traveltime tomography and integralbased Kirchhoff imaging.

For isotropic media, the traveltimes analytically as a function of offset and midpoint is given by a simple double-square-root (DSR) equation in homogeneous media. However, it is difficult to obtain the analytical traveltime formulations in anisotropic media, since the explicit relation between group velocity and ray angle does not exist in anisotropic media. Even for transversely isotropic media with a vertical symmetry axis (VTI), the traveltime is often calculated numerically. Alkhalifah (2000b) derived the offset-midpoint traveltime equation or Cheop's pyramid equation for VTI media through the stationary phase method. Hao and Alexey (2013) extended the offsetmidpoint traveltime equation to the case of $2 \mathrm{D}$ transversely isotropic media with a tilt symmetry axis (TTI).

In this abstract, we derive the analytical offset-midpoint traveltime pyramid for 3D transversely isotropic media with a horizontal symmetry axis (HTI). We specifically obtain a Taylor's series expansion of the slowness components as a function of anelliptic parameter. The accuracy of these expansions is enhanced using Shanks transformation (Bender and Orszag 1978) to obtain the high-order representation.
Time- and depth- domain azimuth-dependent offsetmidpoint traveltime pyramid

The 3D pre-stack phase-shift migration operator defined in the offset-midpoint domain is given by (Yilmaz, 2001),

$$
P\left(x_{1}, x_{2}, h_{1}=0, h_{2}=0, z, t=0\right)
$$$$
=\int d \omega \tilde{P}\left(x_{1}^{0}, x_{2}^{0}, h_{1}^{0}, h_{2}^{0}, z=0, \omega\right) \int d k_{h 1} \int d k_{h 2} \int d k_{x 1} \int d k_{x 2} \exp (-i \omega T)
$$

where $T$ is the traveltime shift given by

$$
\begin{aligned}
T & =\left(q_{s}+q_{g}\right) z+2 p_{x 1}\left(x_{1}-x_{1}^{0}\right) \\
& +2 p_{x 2}\left(x_{2}-x_{2}^{0}\right)-2 p_{h 1} h_{1}^{0}-2 p_{h 2} h_{2}^{0}
\end{aligned},
$$

and $\tilde{P}$ and $P$ are the seismic data before and after prestack depth migration, respectively; $\left(x_{1}^{0}, x_{2}^{0}\right)$ is the lateral position vector of midpoint, $\left(x_{1}, x_{2}\right)$ is the lateral location vector of image point, $z$ is the depth of image point. $\left(h_{1}^{0}, h_{2}^{0}\right)$ is the half-offset vector, $\left(q_{s}, q_{g}\right)$ are the vertical projections of the slowness vector defined at source and receiver positions, respectively, and $\left(p_{x 1}, p_{x 2}\right)$ and $\left(p_{h 1}, p_{h 2}\right)$ are the horizontal slowness vector defined in midpoint-offset space.

Alkhalifah (2000a) used the stationary phase method to calculate the horizontal slowness component at stationary point by solving equation (1) for source slowness $p_{s}$ and receiver slowness $p_{g}$ instead of $p_{h}$ and $p_{x}$, according to the following linear relation,

$$
\begin{aligned}
& p_{s 1}=p_{x 1}-p_{h 1}, \\
& p_{s 2}=p_{x 2}-p_{h 2}, \\
& p_{g 1}=p_{x 1}+p_{h 1}, \\
& p_{g 2}=p_{x 2}+p_{h 2},
\end{aligned}
$$

Hence, equation (1) can be written in terms of slowness for the source and receiver given by,

$$
\begin{aligned}
& T\left(x_{1}, x_{2}, x_{1}^{0}, x_{2}^{0}, h_{1}^{0}, h_{2}^{0}, z\right) \\
& \quad=\left(q_{s}+q_{g}\right) z+p_{s 1} y_{s 1}+p_{s 2} y_{s 2}+p_{g 1} y_{g 1}+p_{g 2} y_{g 2},
\end{aligned}
$$

where $\left(y_{s 1}, y_{s 2}\right)$ denotes the lateral distance vector between image point and source given by $\left(y_{s 1}, y_{s 2}\right)=\left(x_{1}-x_{1}^{0}+h_{1}^{0}, x_{2}-x_{2}^{0}+h_{2}^{0}\right) \quad$ and $\quad\left(y_{g 1}, y_{g 2}\right)$ denotes the lateral distance between image point and receiver given by $\left(y_{g 1}, y_{g 2}\right)=\left(x_{1}-x_{1}^{0}-h_{1}^{0}, x_{2}-x_{2}^{0}-h_{2}^{0}\right)$.

By setting derivatives of travletime $T$ in equation (2) with respect to $p_{s 1}, p_{s 2}, p_{g 1}$ and $p_{g 2}$ to zero, we obtain the equations of the type,

$$
\frac{d q}{d p_{1}}=-\frac{y_{1}}{z}
$$




\section{The azimuth-dependent offset-midpoint traveltime pyramid in 3D HTI media}

$$
\frac{d q}{d p_{2}}=-\frac{y_{2}}{z}
$$

where $\left(p_{1}, p_{2}\right), q$ and $\left(y_{1}, y_{2}\right)$ are either $\left(p_{s 1}, p_{s 2}\right), q_{s}$ and $\left(y_{s 1}, y_{s 2}\right)$ for the source or $\left(p_{g 1}, p_{g 2}\right), q_{g}$ and $\left(y_{g 1}, y_{g 2}\right)$ for the receiver, respectively.

Equations (2), (3) and (4) represent the 3D version of depth-domain traveltime pyramid. In order to obtain the time-domain traveltime pyramid for HTI media, zero-offset two-way traveltime $\tau$ is obtained by setting the half offset vector $\left(h_{1}^{0}, h_{2}^{0}\right)=0$ and lateral coordinate of image point equal to lateral midpoint coordinate, $\left(x_{1}, x_{2}\right)=\left(x_{1}^{0}, x_{2}^{0}\right)$ in equation (2),

$$
\tau=T\left(x_{1}, x_{2}, x_{1}^{0}=x_{1}, x_{2}^{0}=x_{2}, h_{1}^{0}=0, h_{2}^{0}=0, z\right)=2 q_{z 0} z,
$$

where

$$
q_{z 0}=\left.\frac{1}{2}\left(q_{s}+q_{g}\right)\right|_{\substack{x_{1}=x_{1}^{0}, h_{1}^{0}=0 \\ x_{2}=x_{2}^{2}, h_{2}^{1}=0}}=\frac{1}{v_{\text {nmo }} \sqrt{1+2 \eta}}
$$

denotes the vertical slowness for the zero-offset seismic ray. Consequently, by substituting equation (5) into equation (2) we obtain the time-domain traveltime pyramid for the acoustic case in HTI media,

$$
\begin{aligned}
T\left(x_{1}, x_{2}, x_{1}^{0}, x_{2}^{0}, h^{0}, \gamma, \tau\right) & =\frac{1}{2} \sqrt{1+2 \eta} v_{n m o} \tau\left(q_{s}+q_{g}\right) \\
& +p_{s 1} y_{s 1}+p_{s 2} y_{s 2}+p_{g 1} y_{g 1}+p_{g 2} y_{g 2}
\end{aligned}
$$

where $\gamma$ denotes the observation azimuth measured from $\mathrm{x}$-axis,

$$
\tan \gamma=h_{2}^{0} / h_{1}^{0},
$$

$h^{0}$ denotes the size of half-offset vector,

$$
h^{0}=\sqrt{\left(h_{1}^{0}\right)^{2}+\left(h_{2}^{0}\right)^{2}} .
$$

Equation (7) can be employed to calculate the azimuthdependent traveltime of reflected acoustic wave in 3D HTI media. The slowness vector $\left(p_{s 1}, p_{s 2}\right)$ and $\left(p_{g 1}, p_{g 2}\right)$ in equation (7) can also be represented in terms of offset and azimuth.

\section{Horizontal and vertical slowness approximation}

In order to obtain the horizontal slowness $\left(p_{1}, p_{2}\right)$ from equations (3) and (4), we consider the slowness surface equation for HTI media, which describes the relation between horizontal slowness $\left(p_{1}, p_{2}\right)$ and the vertical slowness $q$. According to Alkhalifah (1998, 2000a), the $3 \mathrm{D}$ slowness surface for an acoustic wave in VTI media is given in the following form,

$$
\begin{aligned}
F_{V T I} & =v_{0}^{2} q_{v}^{2}\left(1-2 \eta v_{n m o}^{2}\left(p_{v 1}^{2}+p_{v 2}^{2}\right)\right) \\
& +(1+2 \eta) v_{n m o}^{2}\left(p_{v 1}^{2}+p_{v 2}^{2}\right)-1=0
\end{aligned},
$$

where $\left(p_{v 1}, p_{v 2}\right)$ and $q_{v}$ are the horizontal and vertical slownesses, respectively. $v_{0}$ and $v_{\text {nmo }}=v_{0} \sqrt{1+2 \delta}$ are the vertical and normal moveout velocities, respectively. $\eta=\frac{\varepsilon-\delta}{1+2 \delta}$ is the anelliptic parameter, where $\varepsilon$ and $\delta$ are the anisotropic parameters (Thomsen, 1986).

The slowness surface in TTI media is obtained by applying two separate simple rotations (Zhu and Dorman, 2000). In Figure 1, the $p_{v 1}, p_{v 2}, q_{v}$ system denotes the slowness in VTI media; the $p_{1}, p_{2}, q$ system denotes the slowness in TTI media, where $p_{v 2}$ is located on the $p_{1}-p_{2}$ plane. In the first rotation, we perform a clockwise rotation using $\theta$ along the $p_{v 2}$ axis. After the first rotation, the $q$ and $q_{v}$ axes are coincide. The second rotation is a clockwise using angle $\phi$ about the $q_{v}$ axis.

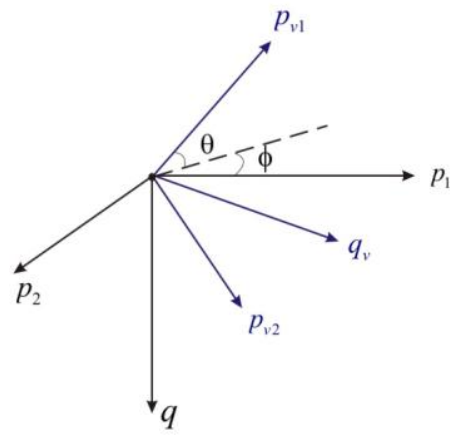

Figure 1: The geometry of slowness vector rotation.

The 3D slowness surface equation for HTI media can be obtained by seting $\theta=\frac{\pi}{2}$,

$$
\left(\begin{array}{c}
p_{1} \\
p_{2} \\
q
\end{array}\right)=\left(\begin{array}{ccc}
0 & \sin \phi & \cos \phi \\
0 & \cos \phi & -\sin \phi \\
-1 & 0 & 0
\end{array}\right)\left(\begin{array}{c}
p_{v 1} \\
p_{v 2} \\
q_{v}
\end{array}\right),
$$

where $\phi$ corresponds to the azimuth of the horizontal symmetry axis measured from the $\mathrm{x}$-axis.

The perturbation of equation (11) can be given in the following form,

$$
\left(\begin{array}{c}
\Delta p_{1} \\
\Delta p_{2} \\
\Delta q
\end{array}\right)=\left(\begin{array}{ccc}
0 & \sin \phi & \cos \phi \\
0 & \cos \phi & -\sin \phi \\
-1 & 0 & 0
\end{array}\right)\left(\begin{array}{c}
\Delta p_{v 1} \\
\Delta p_{v 2} \\
\Delta q_{v}
\end{array}\right),
$$

and the perturbation of the vertical slowness $q$ and $q_{v}$ can be expressed by the corresponding horizontal slownesses, respectively,

$$
\Delta q=\frac{\partial q}{\partial p_{1}} \Delta p_{1}+\frac{\partial q}{\partial p_{2}} \Delta p_{2}
$$




\section{The azimuth-dependent offset-midpoint traveltime pyramid in 3D HTI media}

$$
\Delta q_{v}=\frac{\partial q_{v}}{\partial p_{v 1}} \Delta p_{v 1}+\frac{\partial q}{\partial p_{v 2}} \Delta p_{v 2} .
$$

In order to obtain the partial derivative relations between VTI and HTI media, we assume that the perturbations of the slowness surface in VTI media is caused by a change in only $p_{v 1}$ not $p_{v 2}$, that is

$$
\Delta p_{v 2}=0 \text {. }
$$

Hence, we obtain the following linear algebra equation from equations (12), (13) and (14),

$$
\left(\begin{array}{ccc}
-1 & 0 & \cos \phi \frac{\partial q_{v}}{\partial p_{v 1}} \\
0 & -1 & -\sin \phi \frac{\partial q_{v}}{\partial p_{v 1}} \\
\frac{\partial q}{\partial p_{1}} & \frac{\partial q}{\partial p_{2}} & 1
\end{array}\right)\left(\begin{array}{c}
\Delta p_{1} \\
\Delta p_{2} \\
\Delta p_{v 1}
\end{array}\right)=0 .
$$

Obviously, the existence of a non-zero solution for equation (15) should satisfy that the determinant of the coefficient matrix is zero. Consequently, we obtain

$$
\frac{\partial q_{v}}{\partial p_{v 1}}=-\frac{1}{\cos \phi \frac{\partial q}{\partial p_{1}}-\sin \phi \frac{\partial q}{\partial p_{2}}} .
$$

For the other case, we assume that the perturbations of the slowness surface in VTI media is caused by a change in only $p_{v 2}$ not $p_{v 1}$. In the similar way, we finally obtain

$$
\frac{\partial q_{v}}{\partial p_{v 2}}=-\frac{\cos \phi \frac{\partial q}{\partial p_{2}}+\sin \phi \frac{\partial q}{\partial p_{1}}}{\cos \phi \frac{\partial q}{\partial p_{1}}-\sin \phi \frac{\partial q}{\partial p_{2}}}
$$

Equations (16) and (17) describe the derivative relations between VTI and HTI media. Similar idea was used for mapping of moveout in TI media (Stovas and Alkhalifah, 2012).

In order to calculate the expression for $p_{v 1}$ and $p_{v 2}$, we consider the following relation,

$$
\begin{aligned}
& p_{v 1}=p_{v} \cos \alpha, \\
& p_{v 2}=p_{v} \sin \alpha,
\end{aligned}
$$

where $p_{v}$ denotes the size of horizontal slowness given by

$$
\frac{d q_{v}}{d p_{v}}=\sqrt{\left(\frac{\partial q_{v}}{\partial p_{v 1}}\right)^{2}+\left(\frac{\partial q_{v}}{\partial p_{v 2}}\right)^{2}}=c,
$$

and the explicit expression for $c$ can be obtained from equations (16) and (17) and $\alpha$ denotes the azimuth of horizontal slowness vector, which could be obtained from equation (16) and (17):

$$
\tan \alpha=\frac{\partial q_{v}}{\partial p_{v 2}} / \frac{\partial q_{v}}{\partial p_{v 1}} .
$$

From equations (10) and (18), we obtain polynomials as a function of the slowness $p_{v}$ and $q_{v}$, independently,

$$
\begin{gathered}
c^{2} v_{0}^{2}\left(-1+2 p_{v}^{2} v_{n m o}^{2} \eta\right)^{3}\left(-1+p_{v}^{2} v_{n m o}^{2}(1+2 \eta)\right)-p_{v}^{2} v_{n m o}^{4}=0 \\
c^{2} q_{v}^{2} v_{0}^{4}-\left(1-q_{v}^{2} v_{0}^{2}\right) v_{n m o}^{2}\left(1+2 \eta-2 q_{v}^{2} v_{0}^{2} \eta\right)^{3}=0
\end{gathered}
$$

Both equations (19) and (20) are quartic equations with respect to variables $p_{v}^{2}$ and $q_{v}^{2}$, respectively. To derive the trial solutions, we use the perturbation of $p_{v}$ and $q_{v}$ with respect to the anelliptic parameter $\eta$,

$$
\begin{aligned}
& p_{v}=p_{v_{-} 0}+p_{v_{-} 1}(2 \eta)+p_{v_{-} 2}(2 \eta)^{2}+\ldots, \\
& q_{v}=q_{v_{-} 0}+q_{v_{-} 1}(2 \eta)+q_{v_{-} 2}(2 \eta)^{2}+\ldots .
\end{aligned}
$$

Substituting trial solution (21) and (22) into equation (19) and (20) respectively, the coefficients $p_{v i}$ and $q_{v i}, i=0,1,2$ can be derived. The exact explicit expression for $p_{v i}$ is given in Hao and Stovas (2013).

Furthermore, Shanks transformation can be employed to improve the accuracy of Taylor series expansion for the horizontal slowness and the vertical slowness in VTI media. The final expressions take the form

$$
\begin{aligned}
& p_{v}=p_{v_{-} 0}+\frac{2 p_{v_{-} 1}^{2} \eta}{p_{v_{-} 1}-2 p_{v_{-} 2} \eta}, \\
& q_{v}=q_{v_{-} 0}+\frac{2 q_{v_{-} 1}^{2} \eta}{q_{v_{-} 1}-2 q_{v_{-} 2} \eta} .
\end{aligned}
$$

Considering equation (11), we obtain the norm of horizontal slowness $p$ and the azimuth of horizontal slowness $\beta$ and vertical slowness $q$ in 3D HTI media, respectively,

$$
\begin{gathered}
p=\sqrt{p_{1}^{2}+p_{2}^{2}}=\sqrt{(\sin \alpha)^{2} p_{v}^{2}+q_{v}^{2}}, \\
\tan \beta=p_{2} / p_{1}=\frac{(\cos \phi \sin \beta) p_{v}-\sin \phi q_{v}}{(\sin \phi \sin \beta) p_{v}+\cos \phi q_{v}}, \\
q=-p_{v} \cos \alpha .
\end{gathered}
$$

\section{Numerical examples}

To illustrate the characteristics of the traveltime pyramid as a function of offset and midpoint, we select a homogeneous HTI model with a horizontal symmetry axis along x-axis. The medium parameters are $v_{0}=2 \mathrm{~km} / \mathrm{s}, \delta=0.1, \eta=0.1$. Figure 2 shows the acquisition system used for calculating the traveltime as a function of midpoint and offset for a given azimuth in 3D HTI media. $x_{0}$ and $h$ are the 


\section{The azimuth-dependent offset-midpoint traveltime pyramid in 3D HTI media}

midpoint and the half offset along the observation azimuth, respectively. The image point is located along the z-axis. The zero-offset two-way vertical traveltime $\tau$ is $3 \mathrm{~s}$ for midpoint $x_{0}=0$.

Figure 3 shows the comparison of traveltimes corresponding to different azimuths extracted from the traveltime pyramids for a common-midpoint $\left(x_{0}=0\right)$, for a common-offset $(h=0)$, and for $\left(x_{0}=h\right)$. All three plots in Figure 3 depict that the traveltime decreases with an increase in azimuth from $0^{0}$ to $90^{\circ}$. In the other words, the traveltime pyramid becomes flatten with an increase in azimuth from $0^{0}$ to $90^{\circ}$. This characteristic becomes much more pronounced when offset and midpoint are far from zero.

Next we investigate the variation of common-offset migration isochrones with the change of the observation azimuth. The model parameters in the first example are adopted again. To obtain the common-offset migration isochrones, we assume that both the half offset $h$ and the traveltime $t$ are constant, representing a location in our data space, and we solve for potential image space contribution locations. For $h=0.5 \mathrm{~km}$ and $t=3 \mathrm{~s}$, Figure 4 shows the migration isochrones corresponding to azimuths

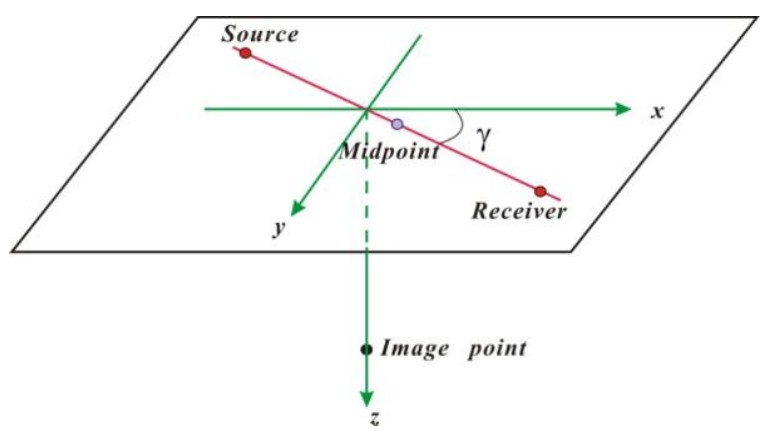

Figure 2: The geometry of the offset-midpoint acquisition system. ranging from $0^{0}$ to $90^{\circ}$. We clearly realize that the azimuthal variations of migration isochrones are much more pronounced for large dip angles where the effect of anisotropy is large. For a horizontal reflector (the isochron apex), all azimuths provide the almost the same placement of energy reflecting the high accuracy of the approximation for this offset.

\section{Conclusions}

The azimuth-dependent offset-midpoint traveltime pyramid for 3D HTI media is derived using the stationary phase method. Perturbation in the anisotropic parameter $\eta$ followed by Shanks transform provides relatively simple analytical formula to describe the traveltimes with high accuracy. The azimuthal characteristic of this traveltime pyramid equation is justifying its potential use in identifying the azimuth of the symmetry axis in 3D HTI media.

\section{Acknowledgements}

We would like to acknowledge the ROSE project for financial support.

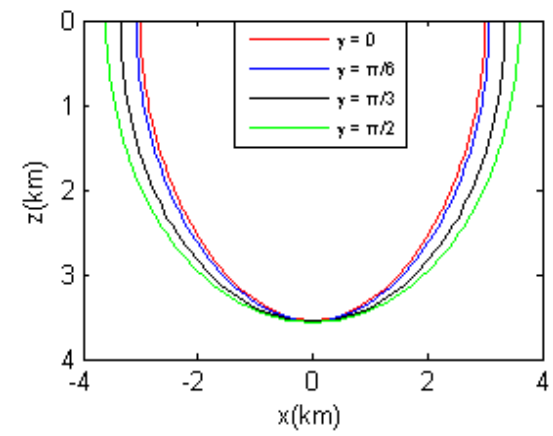

Figure 4: Comparison of common-offset migration isochrones along different observation azimuth.
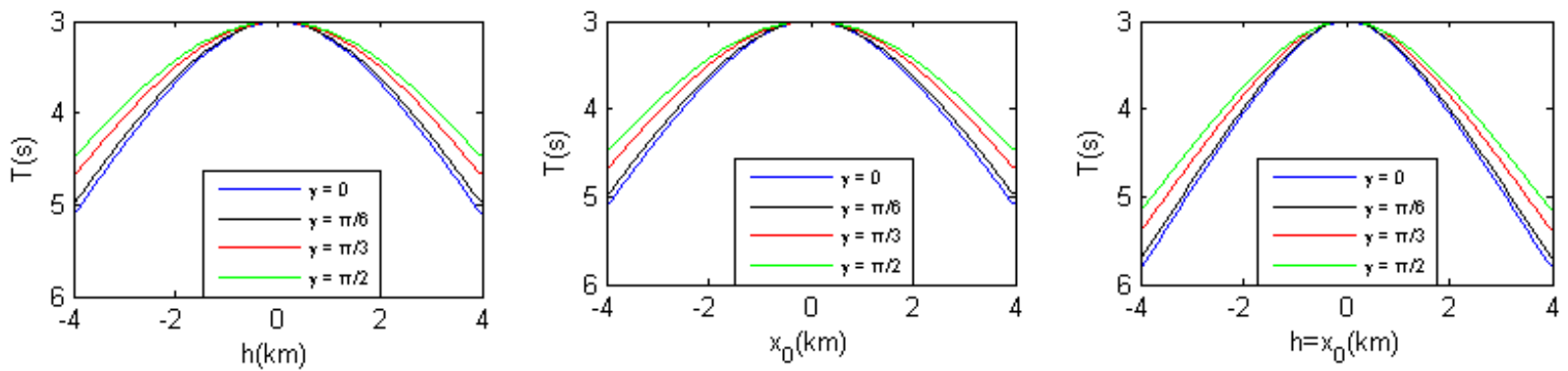

Figure 3: Comparison of azimuth-dependent traveltimes extracted from traveltime pyramids for $\mathrm{x}_{0}=0(\mathrm{left}), \mathrm{h}=0$ (middle) and $\mathrm{x}_{0}=\mathrm{h}$ (right) 
http://dx.doi.org/10.1190/segam2013-0058.1

\section{EDITED REFERENCES}

Note: This reference list is a copy-edited version of the reference list submitted by the author. Reference lists for the 2013 SEG Technical Program Expanded Abstracts have been copy edited so that references provided with the online metadata for each paper will achieve a high degree of linking to cited sources that appear on the Web.

\section{REFERENCES}

Alkhalifah, T., 1998, Acoustic approximations for seismic processing in transversely isotropic media : Geophysics, 63, 623-631, http://dx.doi.org/10.1190/1.1444361.

Alkhalifah, T., 2000a, An acoustic wave equation for anisotropic media : Geophysics, 65, 1239-1250, http://dx.doi.org/10.1190/1.1444815.

Alkhalifah, T., 2000b, The offset-midpoint traveltime pyramid in transversely isotropic media : Geophysics, 65, 1316-1325, http://dx.doi.org/10.1190/1.1444823.

Bender, C. M., and S. A. Orszag, 1978. Advanced mathematical methods for scientists and engineers: McGraw-Hill.

Hao, Q. and A. Stovas, 2013, The offset-midpoint traveltime pyramid in TTI media: Presented at the $75^{\text {th }}$ Annual International Conference and Exhibition, EAGE.

Stovas, A. and T. Alkhalifah, 2012a, A tilted transversely isotropic slowness surface approximation: Geophysical Prospecting, in press.

Stovas, A., and T. Alkhalifah, 2012b, Mapping of moveout in a TTI medium: Presented at the $75^{\text {th }}$ Annual International Conference and Exhibition, EAGE.

Thomsen, L., 1986, Weak elastic anisotropy: Geophysics, 51, 1954-1966, http://dx.doi.org/10.1190/1.1442051.

Yilmaz, O., 2001, Seismic data analysis, processing, inversion and interpretation of seismic data: SEG.

Zhu, J., and J. Dorman, 2000, Two-dimensional, three-component wave propagation in a transversely isotropic medium with arbitrary-orientation-finite-element modeling: Geophysics, 65, 934-942, http://dx.doi.org/10.1190/1.1444789. 